\title{
Classical limit and quantum logic
}

\author{
Marcelo Losada \\ CONICET - Universidad de Buenos Aires, Argentina \\ Sebastian Fortin \\ CONICET - Departamento de Física, \\ Universidad de Buenos Aires, Argentina \\ Federico Holik \\ CONICET - Instituto de Física, Universidad Nacional de La Plata, Argentina
}

(Dated: January 2017)

\begin{abstract}
The analysis of the classical limit of quantum mechanics usually focuses on the state of the system. The general idea is to explain the disappearance of the interference terms of quantum states appealing to the decoherence process induced by the environment. However, in these approaches it is not explained how the structure of quantum properties becomes classical.

In this paper, we consider the classical limit from a different perspective. We consider the set of properties of a quantum system and we study the quantum-to-classical transition of its logical structure. The aim is to open the door to a new study based on dynamical logics, that is, logics that change over time. In particular, we appeal to the notion of hybrid logics to describe semiclassical systems. Moreover, we consider systems with many characteristic decoherence times, whose sublattices of properties become distributive at different times.
\end{abstract}




\section{INTRODUCTION}

The problem of explaining how the laws of classical mechanics arise from the laws of quantum mechanics is known as the classical limit problem [1]. Usually, the description of the classical limit of a quantum system focuses on the state of the system. The idea is to explain the disappearance of the interference terms of quantum states appealing to the decoherence process induced by the environment [2]. Generally, the mathematical description of this process is based on the Schrödinger picture, in which states evolve over time, while observables and physical properties are taken to be constants. As a result, the structure of quantum properties remains the same for all times: the quantum logic associated with the system does not change [3]. Therefore, in these approaches it is not explained how the structure of quantum properties becomes classical.

The properties of a classical system are represented by subsets of the phase space. The set of all properties has a Boolean structure, i.e. it is an orthocomplemented and distributive lattice. This structure is usually called classical logic. Instead, in quantum mechanics the properties are represented by closed vector subspaces or by their corresponding orthogonal projectors [4]. The set of all quantum properties is also an orthocomplemented lattice, but a non-distributive one. This structure is called quantum logic [5]. In this paper, we will argue that the description of the classical limit in terms of the Schrödinger picture is inadequate, because it cannot explain the quantum-to-classical transition of the logical structure of physical properties. If a quantum system undergoes a physical process such that its behaviour becomes classic, then its logical structure of properties should undergo a transition from quantum logic to classical logic, i.e. its lattice structure should become distributive.

In order to give an adequate description of the logical structure transition, we propose to study the classical limit in terms of the Heisenberg picture, in which observables and physical properties evolve over time. This perspective allows to consider the time evolution of the whole lattice of properties. On this basis, we can study the classical limit from a logical point of view, by describing the manner in which the structure of properties becomes a Boolean lattice. The aim is to open the door to the study of dynamical logics, i.e., logics that change over time. In particular, we appeal to the notion of hybrid logics to describe semiclassical systems. Moreover, we consider systems with many characteristic decoherence 
times, whose sublattices of properties become distributive at different times.

The paper is organized in the following way. In Section II, we introduce the logical structure of classical and quantum systems, and we discuss the principal difference between them: the distributive property. In addition, we describe how can be characterized the classical limit from a logical point of view. In Section III, we present different approaches to decoherence and we explain how it is usually related with the classical limit process. Moreover, we argue that the description of the classical limit in terms of the Schrödinger picture is inadequate, because it cannot explain the quantum-to-classical transition of the logical structure of physical properties. In order to achieve a time evolution of the logical structure, we propose to use the Heisenberg picture. In Section IV, we describe the general aspects of the logical perspective of the classical limit process. We apply this approach to describe the quantum-to-classical transition in three different cases: (i) systems with one characteristic time; (ii) transitions from quantum systems to semiclassical systems, in which the quantum logic evolves to a hybrid semiclassical logic; and (iii) systems with many characteristic times, whose sublattices become distributive at different times. Finally, in the last section, we present some conclusions and future perspectives.

\section{LOGICAL STRUCTURE OF QUANTUM MECHANICS}

When we talk about the logical structure of classical mechanics or quantum mechanics, we do not refer to the logic which is used to formulate the theory (both of them are formulated in classical logic), but to the logical structure of the propositions about a classical system or a quantum system.

The propositions about a classical system are determined by classical mechanics and the propositions about a quantum system are determined by quantum mechanics. The way in which classical and quantum mechanics define the set of propositions of a system is by means of the set of properties of the system. Each property $p$ of a system $S$ is associated with the following proposition about the system: the system $S$ has the property $p$.

There is an isomorphism between the set of propositions and the set of properties. Each logical operation between propositions corresponds to an algebraic operation between the corresponding properties. Due to this isomorphism, it is customary to use the term "logical structure" to refer to both structures: propositions structure and properties structure. 
The sets of properties of classical and quantum systems have an orthocomplemented lattice structure. This implies that there is an order relation $\leq$ such that for all pair of properties exists the infimum $\wedge$ and the supremum $\vee$, and all property $p$ has a complement $p^{\perp}$ with adequate properties. All orthocomplemented lattices satisfy certain relations, called distributive inequalities [6]:

$$
\begin{aligned}
& a \wedge(b \vee c) \geq(a \wedge b) \vee(a \wedge c), \\
& a \vee(b \wedge c) \geq(a \vee b) \wedge(a \vee c)
\end{aligned}
$$

When the equalities hold, the lattice is distributive. An orthocomplemented and distributive lattice is called a Boolean lattice. The distributive property is an essential feature which differentiates classical and quantum lattices of properties.

In classical mechanics a physical system is represented by a phase space and the properties of the system are represented by the subsets of its phase space. The partial order relation is given by the inclusion $\subseteq$ of sets. The infimum and the supremum are the intersection $\cap$ and the union $\cup$ of sets, respectively; and the complement of a property $p$ is the complement of sets $p^{c}$. The set of classical properties is not only an orthocomplemented lattice, but also a distributive one, i.e., classical properties satisfy the distributive equalities. Therefore, the logical structure of a classical system is Boolean.

The quantum case is very different. In quantum mechanics a physical system is represented by a Hilbert space and each physical observable is represented by a self-adjoint operator on the Hilbert space [4, 7]. The spectral theorem states that any self-adjoint operator $\hat{A}$ can be represented by its projective measure $M_{A}$ [8-10]. A projective measure assigns an orthogonal projection operator to each Borel set of the real line: given the interval $I(a, b), M_{A}(I)$ is an orthogonal projection operator. This mathematical fact was interpreted by Birkhoff and von Neumann as follows [5]: the projector $M_{A}(I)$ represents the property of having the value of the observable represented by $\hat{A}$ inside the interval $I$. Hence, according these authors, the properties of a quantum system are represented by orthogonal projectors, or by its corresponding closed vector subspaces; and the logical structure of quantum systems is the algebraic structure of closed vector subspaces. This structure is called quantum logic.

Birkhoff and von Neumann developed the main features of quantum logic and they showed its differences with the classical lattice of properties [5]. Then, later developments on the 
logical structure of quantum mechanics were made by several authors (Varadarajan [11], Stubbe and Van Steirteghem [12]; see also Holik et al. [13-15] for more recent developments and for the relationship between the quantum-logical approach and quantum probability theory).

Choosing vector subspaces for representing properties instead of subsets introduces crucial differences in the definition of the algebraic operations of the lattice. As in the classical case, the set of quantum properties has a partial order relation given by the inclusion of subspaces and the infimum is given by the intersection of subspaces. However, the supremum and the complement of properties are different from classical ones. The supremum is given by the sum of subspaces and the complement of a property is its orthogonal subspace [3]. The resulting lattice of quantum properties is orthocomplemented, but it is not distributive [6]. Therefore, quantum logic is not Boolean. This implies a very deep structural difference between classical and quantum theories.

The distributive inequalities are the principal difference between classical and quantum logic. In the classic lattice, all properties satisfy the distributive equalities, but in the quantum lattice, only distributive inequalities hold in general. However, for some subsets of quantum properties the equalities hold. When a subset of properties satisfies the distributive equalities, they are called compatible properties. It can be proved that a sufficient and necessary condition for a set of properties to be compatible is that the projectors associated with the properties commute. Moreover, it can be shown that properties associated with different observables are compatible if the observables commute. If, on the contrary, two observables do not commute, some of the properties associated with them are not compatible. Therefore, by extension, commuting observables are called compatible observables.

The differences between classical and quantum logic are of fundamental importance for the classical limit problem. If a quantum system undergoes a physical process such that its behaviour becomes classic, then its logical structure of properties should undergo a transition from quantum logic to classical logic, i.e. its lattice structure should become distributive.

However, the description of the classical limit of a quantum system usually focuses on the state of the system. The mathematical description of this process is based on the Schrödinger picture, in which states evolve over time, while observables and physical properties are taken to be constants. As a result, the logical structure remains the same for all time. Therefore, in these approaches it is not possible to describe how the structure of quantum properties 
becomes classical.

In order to give an adequate description of the logical structure transition, we propose to study the classical limit in terms of the Heisenberg picture, in which observables and physical properties evolve over time. This perspective allows to consider the time evolution of the whole lattice of properties. On this basis, we can study the classical limit from a logical point of view by describing the manner in which the structure of properties becomes a Boolean lattice.

It is important to remark that, according to Schrödinger equation, a closed system always evolves unitarily. Even in the Heisenberg picture, if two observables are incompatible at one time, they will remain incompatible at any time. Therefore, for describing the quantumto-classical transition of the logical structure, it is necessary to consider non-unitary time evolutions.

In Section 4, we will consider transitions between logics induced by non-unitary time evolutions. Although transitions of these structures were studied before [16], they have not been related with the decoherence process and the classical limit. We think this perspective leads to a better understanding of the classical limit process and it is also useful to deal with hybrid systems.

\section{QUANTUM DECOHERENCE AND OBSERVABLES}

One important feature of the quantum formalism is the superposition principle, which implies the existence of quantum interference phenomena. Any attempt to describe the quantum-to-classical transition should include a mechanism responsible for the suppression of interference.

The usual approach to this problem is based on the evolution of quantum states. The general idea is to show that the interference terms of quantum states disappear when the interaction with the environment is taken into account. This process, known as quantum decoherence, was introduced by H. Dieter Zeh [17, 18] in the early 1970s, and then it was developed by the group led by Wojciech Zurek [19-21]. As decoherence is based on the interaction between the system, considered as an open system, and the environment, it is usually called Environment Induced Decoherence (EID).

In this approach, we consider a closed system $U$ and we divide it into two subsystems: 
the system of interest $S$ and its environment $E$. If the state of the system $U$ is represented by the density operator $\hat{\rho}_{U}(t)$, the state of the subsystem $S$ is represented by the reduced density operator $\hat{\rho}_{S}(t)=\operatorname{Tr}_{E}\left(\hat{\rho}_{U}(t)\right)$ and the state of the environment $E$ is represented by the reduced density operator $\hat{\rho}_{E}(t)=\operatorname{Tr}_{S}\left(\hat{\rho}_{U}(t)\right)$. According to the Schrödinger picture, the closed quantum system $U$ evolves unitarily if no measurements are performed. However, the subsystem $S$ may undergo a non-unitary evolution. This allows that under certain conditions the state $\hat{\rho}_{S}(t)$ becomes diagonal after a decoherence time $t_{D}$. Some authors interpret this process as the essence of the classical limit.

However, it should be emphasized that, in EID approach, quantum decoherence is described from the point of view of the Schrödinger picture. In this representation, the commutator between two observables does not change during the decoherence process. The fact that the commutator between two observables vanishes indicates that those observables are compatible. If, on the contrary, the commutator is not zero, the observables are incompatible and the corresponding properties cannot be included in the same Boolean sublattice. In Schrödinger representation, if two observables are incompatible at the beginning of the process of decoherence, they will remain incompatible during the entire process. Therefore, this approach cannot explain how the structure of quantum properties becomes classical. For this reason, we consider that it is not completely adequate for describing the classical limit process of a physical system.

Alternative approaches to EID have been proposed in order to deal with quantum decoherence in closed systems [22-27]. One of them is the self-induced decoherence approach (SID), developed from the physical and philosophical point of view in several papers [28-35]. In this approach, decoherence is described from the viewpoint of observables. Instead of using Schrödinger picture, SID uses Heisenberg picture, in which observables evolve over time. According to SID, a closed quantum system with continuous spectrum may undergo a decoherence process due to destructive interference. The central point of this proposal consists in a shift of the perspective: instead of splitting the closed system into an open system and an environment, the division is traced between relevant and irrelevant observables. Then, the idea is to analyze the time evolution of the mean values of the relevant observables. The vanishing of the interference terms is interpreted as the result of a decoherence process.

It is important to remark that, according to Schrödinger equation, a closed system always evolves unitarily. Therefore, even in the Heisenberg picture, if two observables $\hat{O}_{1}$ and $\hat{O}_{2}$ do 
not commute at the beginning of the process, they will not commute at any time. This can be expressed in terms of a new observable [36], given by $\hat{C}=i\left[\hat{O}_{1}, \hat{O}_{2}\right]$. We can interpret this observable as measuring the degree of compatibility between $\hat{O}_{1}$ and $\hat{O}_{2}$ : if $\hat{C}=0$, the observables are compatible; if $\hat{C} \neq 0$, they are not.

As a concrete example, in a Mach-Zender interferometer, if $\hat{O}_{1}$ is the observable that measures which is the path taken by the photon and $\hat{O}_{2}$ is the observable associated with the visibility of interference, then $\hat{C}$ can be conceived as the tool to measure how compatible those observables are. In the lab, there are different observables associated with the degree of classicality; for example, the contrast of the interference fringes in the double slit experiment. When the experiment is performed and decoherence occurs, it is reasonable to expect that at the beginning $\hat{C} \neq 0$, but then, after the decoherence time, the observable evolves to $\hat{C}=0$. And it is also expected that, in that limit, the interference fringes will vanish. Moreover, in an experiment with slow and controlled decoherence, it could be possible to measure the evolution of the observable $\hat{C}$.

According to quantum mechanics, a closed system evolves following the Schrödinger equation. Since the evolution is unitary, it is impossible to obtain the following process:

$$
\hat{C}(t=0) \neq 0 \longrightarrow \hat{C}(t)=0 .
$$

However, it has been proved that applying SID approach to systems with continuous energy spectrum, and choosing suitable relevant observables, a similar transition can be obtained, but in terms of the mean value [36]:

$$
\langle\hat{C}(t=0)\rangle \neq 0 \longrightarrow \lim _{t \rightarrow \infty}\langle\hat{C}(t)\rangle=0
$$

Given the incompatible observables $\hat{O}_{1}$, with core $O_{1}(\omega, \tilde{\omega})$, and $\hat{O}_{2}$, with core $O_{2}(\omega, \tilde{\omega})$, both with continuous spectrum, we can compute the commutator $\hat{C}$ as follows:

$$
\hat{C}(t)=i \int_{0}^{\infty} \int_{0}^{\infty} \int_{0}^{\infty}\left(O_{1}(\omega, \tilde{\omega}) O_{2}\left(\tilde{\omega}, \omega^{\prime}\right)-O_{2}(\omega, \tilde{\omega}) O_{1}\left(\tilde{\omega}, \omega^{\prime}\right)\right) e^{i\left(\omega-\omega^{\prime}\right) t} \hat{E}_{\omega, \omega^{\prime}} d \tilde{\omega} d \omega d \omega^{\prime}
$$

where $\left\{\hat{E}_{\omega, \omega^{\prime}}\right\}$ is the energy basis of the space of operators. If $O_{1}(\omega, \tilde{\omega})$ and $O_{2}(\omega, \tilde{\omega})$ are regular functions, then, appealing to the Riemann-Lebesgue theorem, it is possible to prove the following transition [37]:

$$
\langle\hat{C}(t=0)\rangle \neq 0 \longrightarrow \lim _{t \rightarrow \infty}\langle\hat{C}(t)\rangle=0
$$


This implies that the mean value of the observable $\hat{C}$, which measures the incompatibility between two observables, goes to zero. Therefore, since SID approach describes decoherence from the point of view of the observables, it may be a useful tool for studying the quantumto-classical transition of observables.

EID and SID approaches are not the only ways to account for non-unitary evolutions in quantum mechanics. Another strategy to transform the unitary evolution of a closed system into a non-unitary evolution has been proposed in the cosmological context. Kiefer and Polarski [27] adopted the Heisenberg picture for the study of the decoherence process of the universe. In this way, the observable associated with the commutator of two observables becomes a function of time. This approach allows to study the time evolution of the commutator of two observables in the cosmological context.

The general idea of Kiefer and Polarski is to consider a time-dependent Hamiltonian. According to the inflation model, there was an accelerated phase of the early universe called inflation; the whole structure of the universe can be traced back to the primordial fluctuations in the inflaton field [38-40]. Because of the expansion of the universe, inflaton fluctuations must be described by a time-dependent Hamiltonian:

$$
\hat{H}(\eta)=\frac{1}{2} \int d k^{3}\left\{k\left[\hat{a}(k) \hat{a}^{\dagger}(k)+\hat{a}^{\dagger}(-k) \hat{a}(-k)\right]+i \frac{a^{\prime}}{a}\left[\hat{a}^{\dagger}(k) \hat{a}^{\dagger}(k)+\hat{a}(-k) \hat{a}(-k)\right]\right\}
$$

where $\eta$ is the conformal time, $\hat{a}(k), \hat{a}^{\dagger}(k)$ are the annihilation operator and the creator operator respectively, and $a$ is the scale factor of the universe. These three elements are time dependent; hence the Hamiltonian $\hat{H}(\eta)$ is not constant in time. Kiefer and Polarski [27] studied the time evolution of the commutator between the general coordinate $\hat{y}(\eta)$ and its conjugate momentum $\hat{p}(\eta)$. They obtained that the following process is possible:

$$
[\hat{y}(0), \hat{p}(0)] \neq 0 \longrightarrow \lim _{\eta \rightarrow \infty}[\hat{y}(\eta), \hat{p}(\eta)]=0
$$

In other words, the commutator between the position and momentum operator vanishes for times longer than the decoherence time. This fact has an important consequence for the logical structure of the model. The initial algebra, generated by $\hat{y}(0)$ and $\hat{p}(0)$, is noncommutative. Therefore, it has associated a non-distributive propositional lattice, revealing the essential features of what Birkhoff and von Neumann called a quantum logic. On the other hand, the final algebra at $\eta \longrightarrow \infty$, generated by $\hat{y}(\eta)$ and $\hat{p}(\eta)$, is a commutative one. Therefore, it has associated a distributive lattice of propositions, revealing its classical 
nature. This physical process provides an interesting example of the quantum-to-classical transition of logical structures.

Finally, it is important to mention another approach to decoherence based in nonHermitian Hamiltonians. In standard quantum mechanics, the Hamiltonian is a self-adjoint operator and its eigenvalues are real numbers. However, there are another formalisms of quantum mechanics which admit non-Hermitian operators [41, 42]. One example is the Time Asymmetric Quantum Mechanics, formulated in terms of the rigged Hilbert space, and developed for describing the radioactive decay of unstable atoms [43]. Other example is the decoherence formulation based on non-unitary evolutions, in which the interaction with the environment is characterized by an effective non-Hermitian Hamiltonian [44]. These generalizations allow non-unitary evolutions for closed systems. The main idea of these approaches was applied to the study of time evolution of quantum logics by Fortin, Holik and Vanni in [45].

\section{THE CLASSICAL LIMIT FROM THE LOGICAL POINT OF VIEW}

All descriptions of the classical limit of a quantum system should explain the quantumto-classical transition of its logical structure. If a quantum system undergoes a physical process such that its behaviour becomes classical, then its logical structure of properties should undergo a transition from quantum logic to classical logic; i.e., its lattice structure should become distributive. Therefore, if we want to describe adequately this process, we should have at hand a time ordered family of logics, starting with a quantum one, and ending up with a classical one.

In order to be able to describe the logical classical limit, let us consider a quantum system that evolves in a non-unitary way and a set of relevant observables represented by self-adjoint operators, $\mathcal{O}=\left\{\hat{O}_{1}, \hat{O}_{2}, \ldots, \hat{O}_{n}\right\}$. We denote $\mathcal{V}(0)$ to the algebra generated by the set $\mathcal{O}$ at time $t=0$. We also assume that some observables of $\mathcal{O}$ are initially incompatible: for some $i$ and $j,\left[\hat{O}_{i}(0), \hat{O}_{j}(0)\right] \neq 0$.

In what follows, we are going to describe the quantum-to-classical transition in three different cases: systems with one characteristic time; semiclassical systems, and systems with many characteristic times. 


\section{A. Systems with one characteristic time}

For quantum systems with only one characteristic time, the logical classical limit is reached in only one step. In this case, the quantum-to-classical transition is given by the following process:

$$
\left[\hat{O}_{i}(0), \hat{O}_{j}(0)\right] \neq 0 \longrightarrow\left[\hat{O}_{i}\left(t_{D}\right), \hat{O}_{j}\left(t_{D}\right)\right]=0, \quad \forall i, j
$$

Equation (2) suggests an interesting connection between this approach and deformation quantization theory. Indeed, the logical classical limit proposed by this paper can be understood as a deformation of a non-commutative algebra and a limit $\hbar \longrightarrow 0$. Thus, equation (2) can be rewritten in the form

$$
\left[\hat{O}_{i}(0), \hat{O}_{j}(0)\right] \neq 0 \longrightarrow\left[\hat{O}_{i}\left(t_{D}\right), \hat{O}_{j}\left(t_{D}\right)\right]=\imath \hbar\left\{O_{i}\left(t_{D}\right), O_{j}\left(t_{D}\right)\right\}+o\left(\hbar^{2}\right), \quad \forall i, j .
$$

We will leave the study of the relation between equations (2) and (3) for future works.

After time $t_{D}$, the evolving operators generate a family of algebras $\mathcal{V}(t)$. If the classical limit is reached successfully, the final algebra $\mathcal{V}\left(t_{D}\right)$ will be a Boolean algebra, because the final set of generating operators will commute. This means that initially incompatible observables become compatible after the decoherence time.

The algebras $\mathcal{V}(t)$ have associated orthomodular lattices $\mathcal{L}_{\mathcal{V}(t)}$. The logical classical limit is expressed by the fact that, while $\mathcal{L}_{\mathcal{V}(0)}$ is a non-distributive lattice, $\mathcal{L}_{\mathcal{V}\left(t_{D}\right)}$ is a Boolean one. In this way, we obtain an adequate description of the logical evolution of a quantum system.

\section{B. Semiclassical systems}

For a quantum system to become completely classical, it is necessary the commutation of all observables of the system. Notwithstanding, if this condition is strictly applied to all cases of classical limit, it leaves no room for the description of systems which have in some aspects a classical behaviour, but in others a quantum behaviour. Important examples, especially for technology, are the transistors or squids [46], which have classical and quantum properties simultaneously. Let us suppose that we go to an electronics store to buy a transistor. The salesman will first find its location in the shelves, then he will take it in order to put it in a bag and finally he will give it to us. From this point of view, the transistor behaves 
classically: it is an object that can be located in space, and it can be manipulated by classical means. However, when connected to a circuit, well-known quantum effects take place on it; for example, the tunnel effect of the electrons inside it. This means that a transistor is an object with some observables with classical behaviour and with other observables with quantum behaviour. This kind of objects are usually known as semiclassical.

Our approach of the classical limit allows us to account for these cases. In the semiclassical situation, the strong condition (2) turns out to be:

$$
\left[\hat{O}_{i}(0), \hat{O}_{j}(0)\right] \neq 0 \longrightarrow\left[\hat{O}_{i}\left(t_{D}\right), \hat{O}_{j}\left(t_{D}\right)\right]=0, \quad \exists i, j
$$

In other words, there are some initially incompatible observables which become compatible after decoherence time, but also there are some incompatible observables which remain incompatible after the characteristic time. From a logical viewpoint, this implies that the lattices of properties associated with this kind of systems are hybrid lattices.

The focus on hybrid lattices is of particular importance, because it is reasonable to suppose that, if successfully developed, quantum computers will be semiclassical systems, represented by hybrid lattices. This is manifested by the fact that some relevant quantum algorithms possess classical and quantum elements in the process of computation [47]. Therefore, hybrid logics might be useful not only for describing the logical structure of a quantum computer, but also for dealing with the problems related with decoherence.

\section{Systems with many characteristic times}

Up to this point we have considered quantum systems that have only one characteristic time. In this way, we explained the transition from a quantum logic to a Boolean or an hybrid logic. But we have not explored in detail the intermediate steps of this transition. One way to do this is to consider systems with several characteristic times.

There are a number of examples of physical systems that reach the classical limit in several steps. From the point of view of the state, this means that its non-diagonal components vanish at different characteristic times [45]. A concrete example of such a system is an harmonic oscillator embedded in a bath of oscillators [44].

From a logical point of view, the existence of several characteristic times implies that the compatibility condition between different observables is fulfilled at different times. This 
condition can be expressed as follows:

$$
\begin{gathered}
{\left[\hat{O}_{1}(0), \hat{O}_{2}(0)\right] \neq 0 \longrightarrow\left[\hat{O}_{1}\left(t_{\alpha}\right), \hat{O}_{2}\left(t_{\alpha}\right)\right]=0,} \\
{\left[\hat{O}_{1}(0), \hat{O}_{3}(0)\right] \neq 0 \longrightarrow\left[\hat{O}_{1}\left(t_{\beta}\right), \hat{O}_{3}\left(t_{\beta}\right)\right]=0,} \\
\vdots \\
{\left[\hat{O}_{n-1}(0), \hat{O}_{n}(0)\right] \neq 0 \longrightarrow\left[\hat{O}_{n-1}\left(t_{D}\right), \hat{O}_{n}\left(t_{D}\right)\right]=0 .}
\end{gathered}
$$

This means that, among all the initially incompatible observables, some become compatible at time $t_{\alpha}$, others become compatible at time $t_{\beta}$, and so on. If the classical limit is reached, at the end of the process all the observables will commute with each other. In terms of the lattice structure, this many-step process implies that the different parts of the lattice become distributive at different times.

\section{CONCLUSIONS}

Since the very beginnings of quantum mechanics, many attempts have been made to recover the laws of classical physics from quantum theory, through a classical limit process. This process must do the job of turning a quantum system, with a non-distributive logical structure, into a classical system, with a classical logic structure.

The quantum-to-classical transition was extensively studied in the physical literature focusing on the state of the system. However, from this perspective observables do not evolve over time. Therefore, the change of the logical structure of the system is not explained, it is merely understood as a jump from a quantum logic in the beginning to a Boolean one at the end.

In this paper, we argued that the description of the classical limit in terms of the Schrödinger picture is not adequate for explaining the quantum-to-classical transition of the logical structure of a system. If a quantum system becomes classic, then its logical structure of properties should undergo a transition from quantum logic to classical logic. In order to give an adequate description of this transition, we described the classical limit in terms of the Heisenberg picture, in which the lattice of properties evolves in time. On this basis, we showed that it is possible to describe how the quantum structure of properties becomes a Boolean lattice. 
Moreover, we discussed the logical structures associated with a system, not only in the initial and final times, but also during the transition process. As an example of this nontrivial logical structure, we presented a physical system with different characteristic times, in which the classical limit is reached through many steps.

Finally, we applied this logical approach to describe the quantum-to-classical transition in three different cases: (i) systems with one characteristic time; (ii) systems that change from a quantum logic to a hybrid semiclassical logic; and (iii) systems with many characteristic times, whose sublattices become distributive at different times.

We consider that the logical perspective presented in this paper can provide a new insight for the understanding of the classical limit process. This approach based on the logical structure of quantum systems does not intend to be exhaustive or complete, but we expect it to be the kick-off for the study of the dynamical aspects of the logical structures of physical systems. In addition, such research might be useful for understanding the general quantum information processing tasks and the new technologies associated with quantum computers, which involve hybrid logics.

\section{Acknowledgments}

This research was founded by CONICET, ANCyPT, the National University of La Plata and the University of Buenos Aires. The authors want to thank Manuel Gadella and Mario Castagnino for interesting discussions.

[1] D. Cohen. An Introduction to Hilbert Space and Quantum Logic. Berlin: Springer-Verlag (1989)

[2] M. Schlosshauer. Decoherence and the Quantum-to-Classical Transition. Berlin: Springer (2007).

[3] J. Bub. Interpreting the Quantum World. Cambridge: Cambridge University Press (1997).

[4] J. von Neumann. Mathematische Grundlagen der Quantenmechanik. Heidelberg: University Press (1932).

[5] G. Birkhoff, and J. von Neumann. "The Logic of Quantum Mechanics." Annals of mathematics 37 (1936) 823-843. 
[6] G. Kalmbach. Orthomodular Lattices. San Diego: Academic Press (1983).

[7] L. Ballentine. Quantum Mechanics. New York: Prentice Hall (1990).

[8] M. Rèdei. Quantum Logic in Algebraic Approach. Dordrecht: Kluwer Academic Publishers (1998).

[9] M. Reed and B. Simon. Methods of Modern Mathematical Physics I: Functional Analysis. New York: Academic Press (1972).

[10] J. Lacki. "The Early Axiomatizations of Quantum Mechanics: Jordan, von Neumann and the Continuation of Hilbert's Program." Archive for History of Exact Sciences 54 (2000) 279-318.

[11] V. Varadarajan. Geometry of Quantum Theory I. Princeton: van Nostrand (1968).

[12] I. Stubbe and B. Van Steirteghem . "Propositional Systems, Hilbert Lattices and Generalized Hilbert Spaces." In Handbook of Quantum Logic Quantum Structures: Quantum Structures, ed. K. Engesser, D.M. Gabbay and D. Lehmann. Amsterdam: Elsevier (2007) 477-523.

[13] F. Holik, C. Massri, A. Plastino and L. Zuberman. "On the Lattice Structure of Probability Spaces in Quantum Mechanics." International Journal of Theoretical Physics 52 (2013) 18361876.

[14] F. Holik, A. Plastino and M. Sáenz. "A Discussion on the Origin of Quantum Probabilities." Annals of Physics 340 (2014) 293-310.

[15] F. Holik and A. Plastino. "Quantum Mechanics: A New Turn in Probability Theory." In Contemporary Research in Quantum Systems, ed. Z. Ezziane. New York: Nova Publishers (2015) 399-414.

[16] D. Aerts, T. Durt and B. Van Bogaert 1993. "Quantum Probability, the Classical Limit and Nonlocality." In Symposium on the Foundations of Modern Physics 1992: The Copenhagen Interpretation and Wolfgang Pauli, ed. K. V. Laurikainen and C. Montonen. Singapore: World Scientific (1993) 35-56.

[17] H. D. Zeh. "On the Interpretation of Measurement in Quantum theory." Foundations of Physics 1 (1970) 6976.

[18] H. D. Zeh. "Toward a Quantum Theory of Observation." Foundations of Physics 3 (1973) 109116.

[19] W. Zurek. "Environment-Induced Superselection Rules." Physical Review D 26 (1982) 18621880.

[20] W. Zurek. "Decoherence and the Transition from Quantum to Classical." Physics Today 44 
(1991) 36-44.

[21] W. Zurek. "Decoherence, Einselection, and the Quantum Origins of the Classical." Reviews of Modern Physics 75 (2003) 715-775.

[22] L. Diósi. "A Universal Master Equation for the Gravitational Violation of Quantum Mechanics." Physics Letters A 120 (1987) 377-381.

[23] G. Milburn. "Intrinsic Decoherence in Quantum Mechanics." Physical Review A 44 (1991) 5401-5406.

[24] G. Casati and B. Chirikov. "Quantum Chaos: Unexpected Complexity." Physica D 86 (1995) 220-237.

[25] D. Polarski and A. Starobinsky. "Semiclassicality and Decoherence of Cosmological Perturbations." Classical and Quantum Gravity 13 (1996) 377-392.

[26] S. Adler. Quantum Theory as an Emergent Phenomenon. Cambridge: Cambridge University Press (2004).

[27] C. Kiefer and D. Polarski. "Why Do Cosmological Perturbations Look Classical to Us?" Advanced Science Letters 2 (2009) 164-173.

[28] M. Castagnino and O. Lombardi. "The Self-Induced Approach to Decoherence in Cosmology." International Journal of Theoretical Physics 42 (2003) 1281-1299.

[29] M. Castagnino and O. Lombardi. "Self-Induced Decoherence: A New Approach" Studies in History and Philosophy of Modern Physics 35 (2004) 73-107.

[30] M. Castagnino and O. Lombardi. "Self-Induced Decoherence and the Classical Limit of Quantum Mechanics." Philosophy of Science 72 (2005) 764-776.

[31] M. Castagnino and O. Lombardi. "Non-Integrability and Mixing in Quantum Systems: On the Way to Quantum Chaos." Studies in History and Philosophy of Modern Physics 38 (2007) 482-513.

[32] M. Castagnino. "The Classical-Statistical Limit of Quantum Mechanics." Physica A 335 (2004) 511-517.

[33] M. Castagnino and A. Ordóñez. "Algebraic Formulation of Quantum Decoherence." International Journal of Theoretical Physics 43 (2004) 695-719.

[34] O. Lombardi and M. Castagnino. "A Modal-Hamiltonian Interpretation of Quantum Mechanics." Studies in History and Philosophy of Science 39 (2008) 380-443.

[35] M. Castagnino and S. Fortin. "New Bases for a General Definition of the Moving Preferred 
Basis." Modern Physics Letters A 26 (2011) 2365-2373.

[36] S. Fortin and L. Vanni. "Quantum Decoherence: a Logical Perspective." Foundations of Physics 44 (2014) 1258-1268.

[37] M. Castagnino and S. Fortin. "Formal Features of a General Theoretical Framework for Decoherence in Open and Closed Systems." International Journal of Theoretical Physics 52 (2011) 1379-1398.

[38] E. Kolb and M. Turner. The Early Universe. Reading MA: Addison-Wesley (1990)

[39] V. Mukhanov. Physical Foundations of Cosmology. Cambridge: Cambridge University Press (2005).

[40] J. Peacock. Cosmological Physics. Cambridge: Cambridge University Press (1999).

[41] A. Bohm, and N. Harshman. "Quantum theory in the rigged hilbert space Irreversibility from causality." In Irreversibility and Causality Semigroups and Rigged Hilbert Spaces, ed. A. Bohm, H. D. Doebner and P. Kielanowski. Berlin: Springer (2007) 179-237.

[42] N. Moiseyev. Non-Hermitian quantum mechanics. Cambridge: Cambridge University Press (2011).

[43] A. Bohm and M. Gadella. Dirac Kets, Gamow Vectors, and Gel'fand Triplets: The Rigged Hilbert Space Formulation of Quantum Mechanics. Berlin: Springer (1989)

[44] M. Castagnino and S. Fortin. "Non-Hermitian Hamiltonians in Decoherence and Equilibrium Theory." Journal of Physics A 45 (2012) 444009.

[45] S. Fortin, F. Holik and L. Vanni. "Non-Unitary Evolution of Quantum Logics." Springer Proceedings in Physics 184 (2016) 219-234.

[46] J. Clarke and A. Braginski. The SQUID Handbook: Fundamentals and Technology of SQUIDs and SQUID Systems, Volume I. Weinheim: Wiley-VCH (2004).

[47] P. Shor. "Polynomial-Time Algorithms for Prime Factorization and Discrete Logarithm on a Quantum Computer." SIAM Journal on Computing 26 (1997) 1484-1509. 